\title{
Mass publication during the COVID-19 pandemic: Too much of a good thing?
}

\author{
Caleb Anderson BS, Christopher J Peterson MS, Jeff A Dennis PhD
}

The COVID-19 pandemic has seen research disseminated at a rapid rate. As the novel coronavirus progressed from epidemic to global pandemic during the first few months of 2020 , there remained a general void of useful information about SARS-CoV-2 and its pathology. To quickly disseminate needed information about the emerging virus, publishers were encouraged to forego traditional paywalls around virus research. Researchers were also encouraged to submit their data (complete or otherwise) to preprint servers like BioRxiv and MedRxiv, early-access platforms that make non-peer-reviewed articles freely available in the public domain. ${ }^{1}$

The response from the global scientific community was unprecedented; in just under 2 years, over 200,000 academic papers have been published about COVID-19 across both journals and preprint servers. Indeed, publication rates during the current pandemic are much higher than for previous emerging infectious diseases, such as SARS and MERS, and acceptance timeframes are shorter than normal. ${ }^{2-5}$ However, this results in a large and somewhat unwieldy body of research that is difficult to filter in terms of quality, and concerns have arisen that the absence of a formal review process for preprint manuscripts, in tandem with relaxed standards of peer review by editors and journals, has made an already saturated research landscape even harder to navigate by allowing research of low or questionable quality into the public domain. These concerns are not unfounded; since the beginning of the pandemic, there have been numerous high-profile studies that attracted significant interest before being retracted.

Corresponding author: Christopher Peterson Contact Information: Christopher.Peterson@ttuhsc.edu DOI: 10.12746/swrccc.v10i42.959
Bibliometric analyses of COVID-19 studies published early in the pandemic noted a high degree of bias in most articles. Many of these demonstrated poor methodological rigor across common design elements like inclusion criteria and adherence to standard reporting frameworks. ${ }^{6}$ The relationship between COVID-19 and smoking is illustrative. In the early months of the COVID19 pandemic, many observational studies reported on the relationship between common risk factors such as diabetes, coronary artery disease (COPD), and coronary artery disease (CAD), and COVID-19 hospitalization. Results were generally as expected; factors such as COPD and obesity were associated with greater disease severity and worse outcomes. However, the association between smoking and disease outcomes was unexpected. Although former smokers were at an increased risk of hospitalization, in-hospital disease severity, and mortality compared to never smokers, current smokers were observed to have a lower risk of testing positive for COVID-19. Subsequent studies investigated the potential effect smoking/nicotine had in the pathogenesis of COVID-19 infection, with some suggesting mechanisms by which smoking could be protective, such as downregulation of ACE-2 receptors found in the lungs and used as an entry receptor for SARS-CoV-2. ${ }^{7}$

Many studies have been published on this topic, including over 25 meta-analyses. One such analysis, published in Addiction in 2021, identified 233 published articles or preprints that report on the association of smoking and COVID-19. ${ }^{8}$ Of these studies, 186 were rated by the authors as being poor in quality. Of the remaining studies, 46 were rated as fair in quality, and 1 study was rated to be of good quality. Relevant to this breakdown is the fact that over 160 of the cited articles in this meta-analysis were preprint articles on the medRxiv site and had not formally been accepted as peer-reviewed publications. Nevertheless, at the time of this writing, Google Scholar indicates that this 
meta-analysis has been cited 164 times. Further, the review has been updated from version 7 (published in Addiction) to an online version 12, which identifies 547 total studies with 9 being of "good" quality. ${ }^{8}$ Lower-quality studies on smoking and COVID-19 often failed to differentiate between current or former smoking status, determine pack-years of smokers, or control for comorbidities. ${ }^{9-13}$ Other noted study flaws and limitations included inappropriate extrapolation to the general population, ${ }^{9}$ lack of statistical significance, ${ }^{14}$ potential differences in health preventive behaviors between smokers and non-smokers, ${ }^{15}$ failure to adjust for confounding variables ${ }^{16}$ incomplete data, selection bias, and misclassification bias. ${ }^{13}$

The publication of so many studies on this topic raises several issues. First, while the initially reported protective effect of smoking on COVID-19 is intriguing, the utility of a possible protective effect of smoking for COVID-19 is doubtful. Even if smokers hypothetically exhibited some type of protection from COVID-19, the increased risks of neoplastic pathologies and cardiovascular damage associated with smoking are such that no amount of cigarette use would be justified toward protection from COVID-19. While the existence of such a paradoxical protective effect would doubtless be intriguing, it is unlikely to change public health response to the pandemic.

Next, both the mass production and dissemination of a large amount of research during the pandemic speaks to the academic community's ability to respond to a public health emergency. In this case, researchers helped dispel unusual and misleading conclusions about smoking and COVID-19. Nevertheless, the utility of multiple, lower-quality studies should be questioned. While multiple studies can contribute to a larger body of knowledge that can be summarized in meta-analyses and reviews, multiple low-quality studies will not aggregate into high-quality data. Furthermore, a large body of information, particularly conflicting data, may lead to so-called "information overload", in which overexposure to information can lead to negative outcomes. ${ }^{17,18}$ Misinformation may also negatively affect health-protective behaviors. For example, a survey in Hong Kong found that exposure to misinformation about smoking/alcohol consumption and COVID-19 resulted in increased rates of tobacco and alcohol consumption. ${ }^{19}$ Finally, a large body of lower quality studies risks the communication of such research to the public, which may negatively impact public confidence in the scientific and medical community. ${ }^{20}$ Whereas the goal of open access publication is to make scientific information more accessible to all, the potential for both intentional and unintentional misinterpretation increases substantially with the proliferation of studies without rigorous peer review.

While research is needed in public health crises, the urgency for information must be balanced with quality research and publication safeguards. Preprint servers and expedited review processes can help research dissemination while simultaneously circulating lower-quality research. Despite the desire for rapid research and evaluation during an infectious disease pandemic, scientific methods and standards must remain rigorous, and findings must remain open to critique, even when this process takes longer than the urgency of the situation might dictate. Though there is no simple solution during extenuating circumstances such as public health crises, researchers and publishers should be aware of the potential negative impact lower quality research can have and recognize that such work can be counterproductive toward the goal of scientific credibility.

Keywords: COVID-19; publication; smoking; information overload

Article citation: Anderson C, Peterson CJ, Dennis JA. Mass publication during the COVID-19 pandemic: Too much of a good thing?. The Southwest Respiratory and Critical Care Chronicles 2022;10(42):22-24

From: School of Medicine (CJP), Department of Internal Medicine (CA), Department of Public Health (JAD), Texas Tech University Health Sciences Center, Lubbock, Texas

Submitted: $12 / 23 / 2021$

Accepted: $1 / 7 / 2022$

Conflicts of interest: none

This work is licensed under a Creative Commons Attribution-ShareAlike 4.0 International License. 


\section{REFERENCES}

1. Sharing research data and findings relevant to the novel coronavirus (COVID-19) outbreak. Wellcome, 2020. At https:// wellcome.org/press-release/sharing-research-data-and-findings-relevant-novel-coronavirus-ncov-outbreak.

2. Yeo-Teh NSL, Tang BL. An alarming retraction rate for scientific publications on Coronavirus Disease 2019 (COVID19). Account Res 2021;28:47-53.

3. Palayew A, Norgaard O, Safreed-Harmon K, et al. Pandemic publishing poses a new COVID-19 challenge. Nature Human Behaviour 2020;4:666-9.

4. Di Girolamo N, Meursinge Reynders R. Characteristics of scientific articles on COVID-19 published during the initial 3 months of the pandemic. Scientometrics 2020:1-18.

5. Else H. How a torrent of COVID science changed research publishing - in seven charts. Nature 2020;588:553.

6. Khatter A, Naughton M, Dambha-Miller H, et al. Is rapid scientific publication also high quality? Bibliometric analysis of highly disseminated COVID-19 research papers. Learn Publ 2021;34(4):568-577

7. He Y, Sun J, Ding X, Wang Q. Mechanisms in which smoking increases the risk of covid-19 infection: a narrative review. Iran J Public Health 2021;50:431-7.

8. Simons D, Shahab L, Brown J, et al. The association of smoking status with SARS-CoV-2 infection, hospitalization and mortality from COVID-19: a living rapid evidence review with Bayesian meta-analyses (version 7). Addiction 2021; 116:1319-68.

9. Wenzl T. Smoking and COVID-19: Did we overlook representativeness? Tobacco Induced Diseases 2020;18:1-2.

10. Alexander PE, Debono VB, Mammen MJ, et al. COVID-19 coronavirus research has overall low methodological quality thus far: case in point for chloroquine/hydroxychloroquine. J Clinical Epidemiology 2020;123:120-6.
11. Jung RG, Di Santo P, Clifford C, et al. Methodological quality of COVID-19 clinical research. Nature Communications 2021;12:943.

12. Yu Y, Shi Q, Zheng P, et al. Assessment of the quality of systematic reviews on COVID-19: A comparative study of previous coronavirus outbreaks. J Med Viro 2020;92: 883-90.

13. van Westen-Lagerweij NA, Meijer E, Meeuwsen EG, et al. Are smokers protected against SARS-CoV-2 infection (COVID-19)? The origins of the myth. Primary Care Respiratory Medicine 2021;31:10.

14. Wenzl T. Smoking and COVID-19- A review of studies suggesting a protective effect of smoking against COVID-19 2020. Report No.: JRC121837.

15. González-Rubio J, Navarro-López C, López-Nájera E, et al. A systematic review and meta-analysis of hospitalised current smokers and COVID-19. International J Environmental Research Public Health 2020;17:7394.

16. Patanavanich R, Glantz SA. Smoking is associated with COVID-19 progression: a meta-analysis. Nicotine Tob Res 2020;22:1653-6.

17. Mohammed M, Sha'aban A, Jatau AI, et al. Assessment of COVID-19 information overload among the general public. J Racial Ethn Health Disparities 2021:1-9.

18. Hong H, Kim HJ. Antecedents and consequences of information overload in the COVID-19 pandemic. International J Environmental Research Public Health 2020;17: 9305.

19. Luk TT, Zhao S, Weng X, et al. Exposure to health misinformation about COVID-19 and increased tobacco and alcohol use: a population-based survey in Hong Kong. Tobacco Control 2020: tobaccocontrol-2020-055960.

20. West JD, Bergstrom CT. Misinformation in and about science. Proceedings National Academy of Sciences 2021;118: e1912444117. 